\title{
Single transluminal gateway transcystic multiple drainage for extensive walled-off pancreatic necrosis - a single-centre experience
}

\author{
Mateusz Jagielski, Marian Smoczyński, Krystian Adrych \\ Department of Gastroenterology and Hepatology, Medical University of Gdansk, Gdansk, Poland
}

Gastroenterology Rev 2018; 13 (3): 242-248

DOI: https://doi.org/10.5114/pg.2018.78290

Key words: walled-off pancreatic necrosis, endoscopic drainage.

Address for correspondence: Mateusz Jagielski MD, PhD, Department of Gastroenterology and Hepatology, Medical University of Gdansk, 17 Smoluchowskiego St, 80-214 Gdansk, Poland, phone: +48 781143 696, e-mail: matjagiel@gmail.com

\begin{abstract}
Introduction: In last three decades we have observed development in minimally invasive techniques in the treatment of walled-off pancreatic necrosis (WOPN). Endoscopic treatment of the consequences of acute necrotising pancreatitis is a accepted and common minimally invasive method.

Aim: Evaluation of the efficiency and safety of the innovative endoscopic treatment method (single transluminal gateway transcystic multiple drainage - SGTMD) in patients with extensive walled-off pancreatic necrosis.

Material and methods: The retrospective analysis of 114 consecutive patients with symptomatic WOPN, who were treated endoscopically in our medical centre between 2011 and 2016.

Results: Single transluminal gateway transcystic multiple drainage was performed in 21/114 (18.42\%) patients. Endoscopic necrosectomy under fluoroscopic guidance was performed in 12/21 (57.14\%) patients. Complications of treatment appeared in $7 / 21$ (33.33\%) patients. The most common of complication was upper gastrointestinal bleeding treated conservatively with packed red blood cells transfusions. There were no deaths. Therapeutic success was reached in 20/21 (95.24\%) patients. No patient required surgery. The medium time of follow-up was 22 months (16-47). During the observation the recurrence of pancreatic fluid collection was noticed in $1 / 21$ (4.76\%) patients. Long-term success of endoscopic treatment was achieved in 19/21 (90.47\%) patients.

Conclusions: Single transluminal gateway transcystic multiple drainage is an effective method of endoscopic treatment for extensive walled-off pancreatic necrosis with an acceptable amount of complications. However, the method of interventional treatment of pancreatic necrosis should depend not only on the location of necrosis, but also on the experience of the medical centre.
\end{abstract}

\section{Introduction}

Pancreatic fluid collections (PFCs) are well-known consequence of moderate and severe acute pancreatitis [1-4]. Acute necrotic collection (ANC) is created in the early phase of acute necrotising pancreatitis (ANP) [2, 3]. Preserved ANC after 4 weeks of ANP is defined as walled-off pancreatic necrosis (WOPN) [2, 3]. According to the revision of the Atlanta classification from 2012, WOPN is PFC surrounded by mature wall and includes liquefied necrosis and necrotic tissues in its lumen [2, 3]. Walled-off pancreatic necrosis is discovered in about $15 \%$ of patients with severe acute pancreatitis [4].

The first descriptions of an efficient endoscopic drainage of PFCs were published in 1980s [5]. In 1992
Grimm et al., as the first in the world, introduced a description of endoscopic drainage of PFCs under control of endoscopic ultrasonography (EUS) [6]. The first publications concerning endotherapy of PFCs referred to pancreatic pseudocysts $[5,6]$, which are a collection including liquefied content only, without any tissues $[2,3]$. Endoscopic drainage/debridement of WOPN was written up for the first time by Baron et al. in 1996 [7]. The results of endoscopic drainage of walled-off pancreatic necrosis are worse than those concerning endotherapy of pseudocysts $[8,9]$, due to dense content and solid necrotic tissues in the lumen of WOPN [1-3]. The patients with WOPN require more aggressive endoscopic treatment [4, 10-12]. Transmural endoscopic 
drainage is a common method of treatment for patients with symptomatic WOPN $[13,14]$.

Single transluminal gateway technique (SGT) is based on complete removal of necrotic tissues through the single fistula created between the cavity of necrotic collection and the lumen of gastrointestinal tract (stomach or duodenum) $[7,13,14]$. The conventional SGT using transmural placement of single or multiple stents and nasocystic drain is insufficient in some patients with WOPN - particularly when it is infected. When single access to necrotic collection is not effective enough, additional access to necrosis is necessary $[4,13]$. Percutaneous drainage is usually performed then [15].

This paper presents our own experience in endoscopic treatment of patients with extensive WOPN and indicates the potential therapeutic possibilities created by using the technique of treatment allowing an access to extensive necrotic areas through a single transmural fistula. Herein we present the results of treatment of 21 patients with symptomatic WOPN, in whom the innovative treatment method (single transluminal gateway transcystic multiple drainage - SGTMD) benefited as the only access route to the necrotic cavity.

\section{Aim}

The retrospective analysis of efficiency and safety of SGTMD in patients with extensive walled-off pancreatic necrosis.

\section{Material and methods}

A total of 114 consecutive patients ( 81 men, 33 women, mean age: 51.7 years (21-85)) with symptomatic WOPN were treated endoscopically in our medical centre between 2011 and 2016. The qualification for endoscopic treatment was based on clinical picture along with abdominal contrast-enhanced computed tomography (CECT). Walled-off pancreatic necrosis was stated according the criteria of revision of Atlanta classification from 2012 [2, 3]. The morphology of liquid (dark-brown colour and fragments of necrotic tissues) aspirated from the lumen of necrotic collection confirmed recognition of WOPN in every patient.

A detailed description of the endoscopic methods applied to the patients in our medical centre was included in our previous publications $[10,13,16,17]$. Transmural drainage was attempted in all patients. The site of fistulotomy was chosen under EUS-guidance. Enterostomy was performed with use of Giovannini's cystostome (Cystotome CST-10, Wilson-Cook). Fistula created between the lumen of the gastrointestinal tract and lumen of necrotic collection was widened with a high-pressure balloon of diameter from $8 \mathrm{~mm}$ to $20 \mathrm{~mm}$ (Boston Scientific). Nasal drain (7 Fr or $8 \mathrm{Fr}$, Bal- tonor Wilson-Cook) as well as double pigtail endoprostheses (7 Fr or $8.5 \mathrm{Fr}$, Wilson-Cook or MarFlow) were inserted through the stomy. Distal ends of the drain and stents were positioned in the lumen of necrotic cavity. Collection of WOPN was flushed with saline solution $(60-200 \mathrm{ml})$ through the nasal drain every $2 \mathrm{~h}$ in the first $48 \mathrm{~h}$ and every $4 \mathrm{~h}$ after that.

In the early phase of the study (2011-2013) additional percutaneous drainage was performed, when endoscopic drainage had become ineffective or when the necrosis had spread outside the lesser omental sac. In the later stage of the study (2013-2016), if drainage through a single transmural access (SGT) was ineffective, another transmural tract was created (multiple transluminal gateway technique - MTGT) when there was no communication between the necrotic collection sub-cavities or multiple access through a single transmural fistula (SGTMD) was used, which involved obtaining additional access to extensive necrotic areas through a single transmural tract (Figures $1 \mathrm{~A}-\mathrm{H}$ ). What is more, endoscopic necrosectomy under fluoroscopic guidance [10] was executed during the transmural drainage in selected patients. Criteria of qualification for endoscopic necrosectomy are: lack of clinical improvement despite the applied drainage, infection of necrotic collection, and discovery of a large amount of necrotic tissues in the fluoroscopic and endosonographic picture [10].

In patients treated with SGTMD (Figures 2 A-D) a guidewire was inserted to the further sub-cavities under fluoroscopic image during subsequent endoscopic procedures. Canals between necrotic areas were widened with use of an 8-mm high-pressure balloon (Boston Scientific) under fluoroscopy (Figure 3). Thereafter, next nasal drain or endoprostheses were guided through the canals to the sub-cavities of WOPN.

The procedure of endoscopic necrosectomy was started with the removal of the nasal drain. The next step was to insert a Dormia basket (FG-V422PR, Olympus) through the fistula to the necrotic collection, leaving the transmural stents. Necrotic tissues were removed with the Dormia basket and under fluoroscopy through transmural fistula. This action was repeated several times during each procedure of necrosectomy. A nasal drain was put in transmurally again after the end of the procedure.

The size of WOPN was controlled by conventional ultrasonography of the abdomen every 7 days in most of the patients. The CECT of the abdomen was done in order to confirm the complete regression of collection.

Endoscopic drainage was ended in the moment of reaching therapeutic success, which was defined as a lack of symptoms and complete regression of collection or size of collection below $40 \mathrm{~mm}$ in the imaging examinations. 

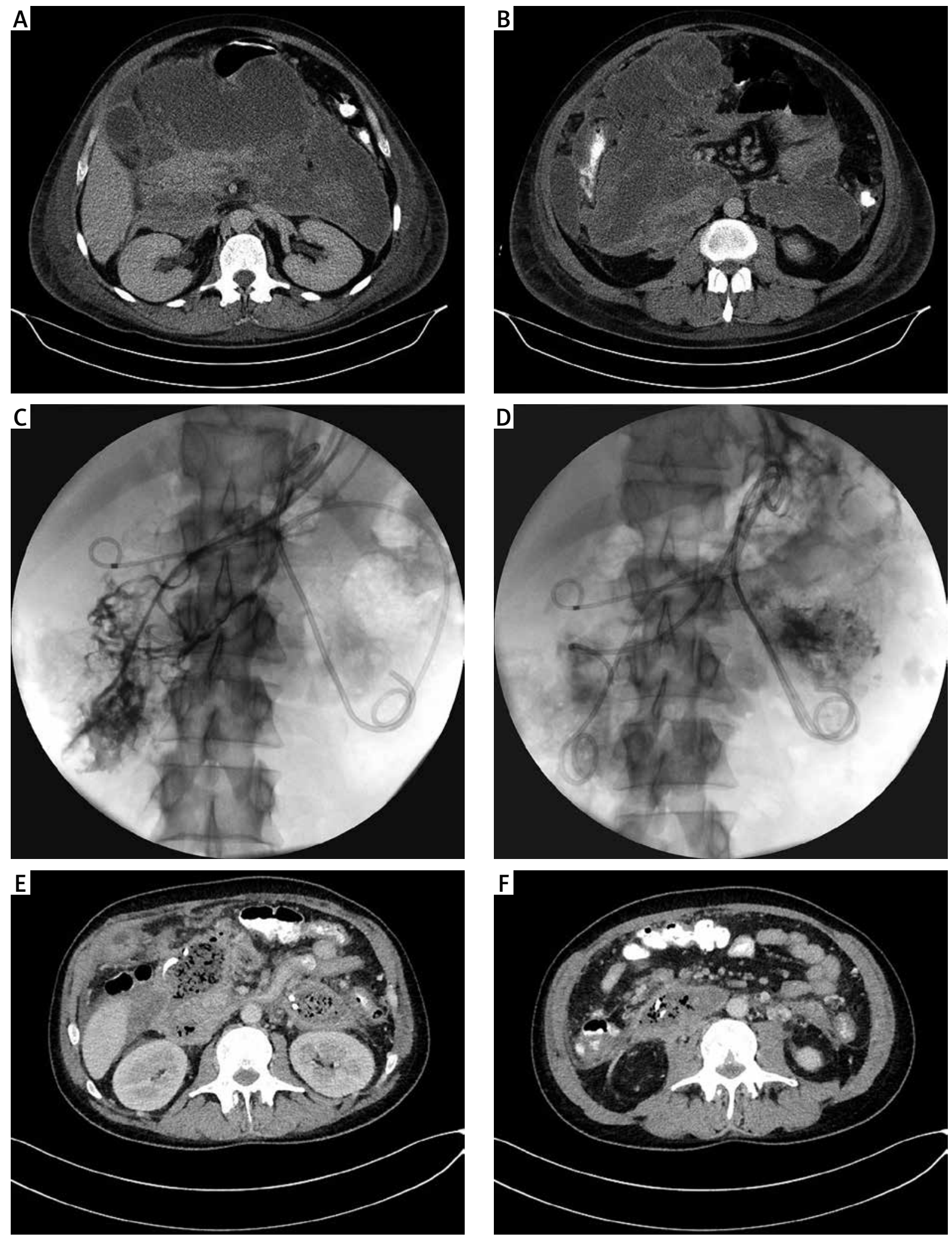

Figure 1. The patient with an extensive walled-off pancreatic necrosis visible in contrast-enhanced computed tomography (A, B). The SGTMD technique was exploited for treatment (C-F). Endoscopic necrosectomy was performed during transmural drainage 

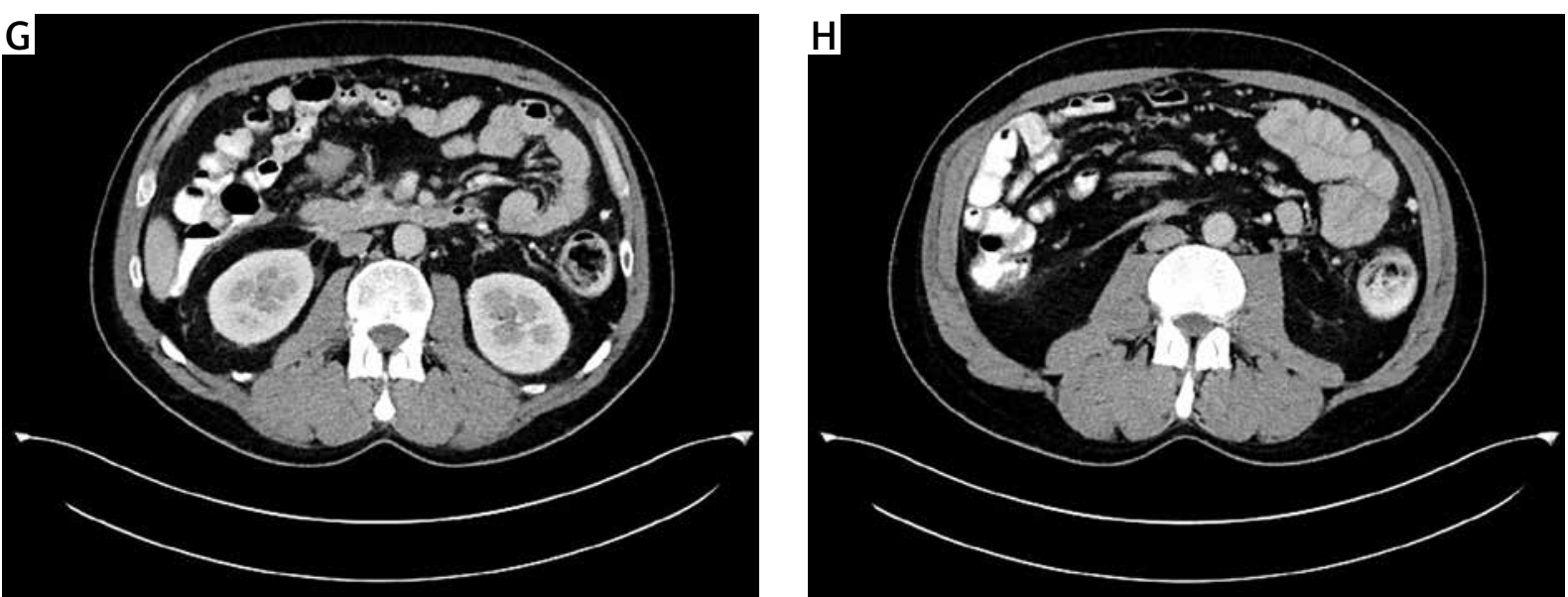

Figure 1. Cont. The CECT performed after the end of endoscopic treatment showed complete regression of WOPN $(\mathbf{G}, \mathrm{H})$
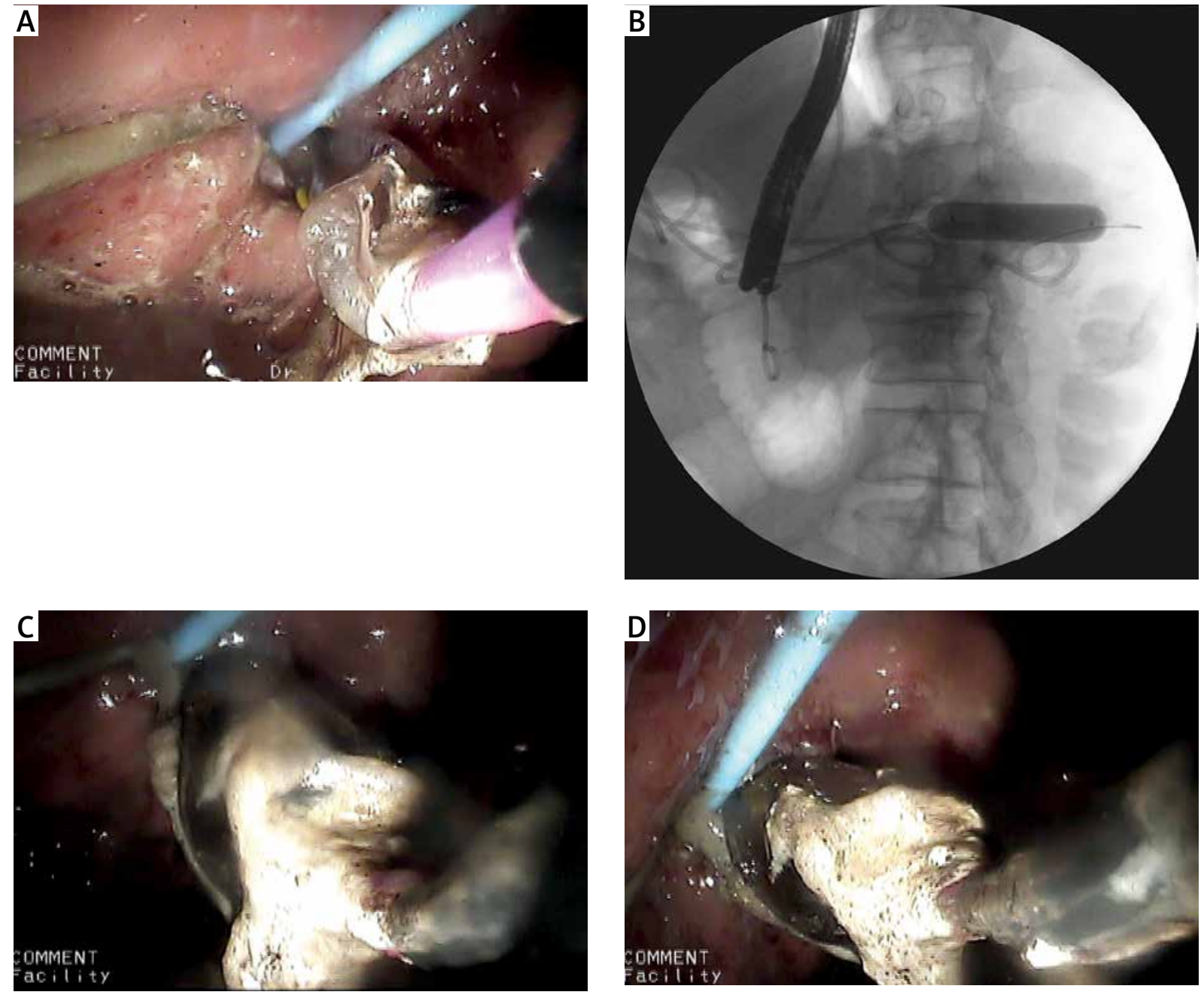

Figure 2. Single transluminal gateway transcystic multiple drainage. Canals between necrotic areas were widened with use of an 8-mm high-pressure balloon under fluoroscopy (A, B). During removal of the balloon through the necrogastrostomy numerous fragments of necrotic tissues were also removed (C, D) 


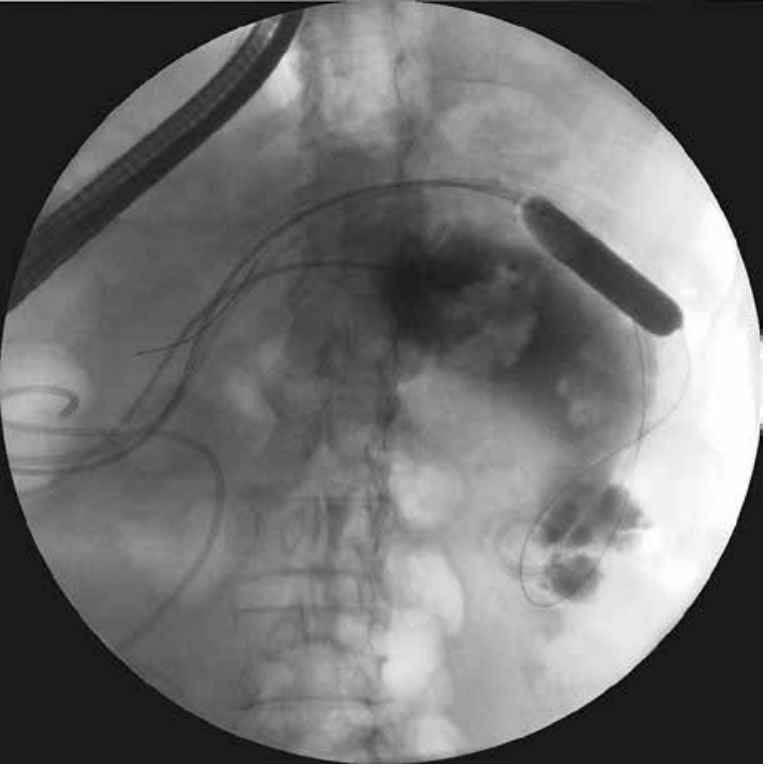

Figure 3. Single transluminal gateway transcystic multiple drainage. Canals between necrotic areas were widened with use of an $8-\mathrm{mm}$ high-pressure balloon under fluoroscopic image

Long-term success was defined as lack of symptoms and complete regression of collection or size of collection below $40 \mathrm{~mm}$ in the imaging examinations during 1 -year observation from the end of active endoscopic drainage. Recurrence of collection was defined as size of collection beyond $40 \mathrm{~mm}$ or recurrence of symptoms during 1-year observation.

\section{Statistical analysis}

All the statistical calculations were performed with the use of data analysis software system StatSoft Inc. (2011) Statistica version 10.0 (licensed for Medical University of Gdansk). Quantitative variables were characterised by arithmetic means, and minimal and maximal values (range), whereas qualitative data were presented by means of numbers and percentages.

\section{Results}

A total of 114 patients with symptomatic WOPN were treated endoscopically in our medical centre between 2011 and 2016. Single transluminal gateway transcystic multiple drainage was performed in 21/114 (18.42\%) patients (14 men, seven women, mean age: 47.76 years (33-81)) (Figures $1 \mathrm{~A}-\mathrm{H})$. The aetiology of acute pancreatitis was alcoholic in $13 / 21$ (61.9\%) patients. The aetiology was non-alcoholic in the remaining 8/21 (38.1\%) patients (5 biliary, 2 iatrogenic, 1 hypertriglyceridaemia). Average time from the acute of pancreatitis until the beginning of endotherapy was
64 days (23-126). The mean size of WOPN was $19.25 \mathrm{~cm}$ (10.0-33.4). Mixed necrosis (pancreatic and peripancreatic necrosis) was confirmed in all 21 patients.

The indications for the beginning of endoscopic WOPN treatment in study group were as follows: gastrointestinal obstruction together with weight loss and abdominal pain (13/21 patients), clinical suspicion of infected WOPN (8/20 patients), and jaundice (1/21 patients).

The WOPN infection was diagnosed on the basis of positive microbial culture in 7/21 (33.33\%) patients, in whom infection was suspected. The most common pathogens cultured in the necrotic contents were Escherichia coli and Enterococcus faecalis. In 4/21 (19.05\%) patients sepsis with positive blood culture (Escherichia coli - 3 patients; Enterococcus faecalis 1 patient) was observed during drainage.

Endoscopic transmural access was used in all 21 patients (gastric - 20, duodenal - 1). Endoscopic necrosectomy under fluoroscopic guidance was performed in $12 / 21(57.14 \%)$ patients.

Complications of treatment appeared in 7/21 (33.33\%) patients. Upper gastrointestinal bleeding treated conservatively with packed red blood cell transfusions was stated in 6 patients. One patient had migration of transmural stent into the lumen of WOPN treated endoscopically. There were no deaths.

Endoscopic retrograde pancreatography (ERP) was done in 20/21 (95.24\%) patients in order to evaluate the main pancreatic duct (MPD). One patient was unable to have ERP done, due to failed MPD cannulation caused by deformation of duodenum and luminal compression making identification of papilla impossible. The leak of contrast outside the MPD during ERP (MPD disruption) took place in $14 / 20$ patients. The fragment of the duct was contrasted without a visible leak in $6 / 20$ patients.

Therapeutic success was reached in 20/21 (95.24\%) patients. One patient still requires passive transmural drainage because of remaining size of pancreatic collection beyond $40 \mathrm{~mm}$ and disconnected duct syndrome. No patient required surgery. The mean time of endoscopic drainage was 34.21 days (20-82). The average number of endoscopic procedures was 5.9 (3-23). The average number of necrosectomy procedures was 7.1 (2-15).

The medium time of follow-up was 22 months (1647). During the observation the recurrence of pancreatic fluid collection (in the form of pancreatic pseudocysts) was noticed in $1 / 21$ (4.76\%) patients. Long-term success of endoscopic treatment was achieved in 19/21 (90.47\%) patients.

\section{Discussion}

This paper presents an innovative method of endoscopic treatment of patients with extensive WOPN 
without the use of other minimally invasive techniques. The basis of therapeutic strategy in this technique of transmural drainage is widening of the access to necrotic areas through single transluminal necrogastrostomy or necroduodenostomy. Our publication shows that single transluminal gateway transcystic multiple drainage is an effective and safe method of treatment in patients with symptomatic pancreatic necrosis. Furthermore, endoscopic necrosectomy under fluoroscopic guidance during transmural drainage improves the results of interventional treatment of selected patients with WOPN.

Development of a minimally invasive method of treatment of pancreatic necrosis [10-18] enabled the design a scheme of therapy of WOPN [4]. The currently accepted strategy in treatment of WOPN (so-called "step-up approach") consist of initial management of symptomatic pancreatic necrosis with antibiotics and nutritional support, followed by minimally invasive drainage (endoscopic, percutaneous, or surgical) if symptoms persist. Endoscopic or surgical necrosectomy should be taken into consideration only if there is no positive response to previously applied treatment. Optimal treatment strategy for symptomatic pancreatic necrosis stands for multiple access to necrotic collection, which can be used with the application of a few minimally invasive methods. Widening of the access to necrotic areas provides better drainage conditions and increases the efficiency of interventional treatment $[4,10,11]$. However, the method of access to WOPN should depend not only on the location of necrosis, but also on the experience of the medical centre [10-12].

Endoscopic treatment of WOPN, like other minimally invasive methods, has evolved. The diameter of transmural fistula has been being increased up to $20 \mathrm{~mm}$ with the popularisation of endotherapy, which enabled insertion of a fiberscope into the cavity of necrosis and performance of endoscopic necrosectomy $[10,14,18,19]$. In our paper endoscopic necrosectomy under fluoroscopic guidance was performed in 12/21 (57.14\%) patients. This technique of endoscopic necrosectomy consisting of the removal of necrotic tissues under fluoroscopic guidance without the need to insert a fiberscope into the cavity of WOPN [10]. This method of necrosectomy as an alternative to other techniques used for treatment of patients after acute necrotic pancreatitis was described in 2015 [10].

Not only has the diameter of fistula increased with the development of endoscopic methods of treatment, but also the number of transmural fistulas. Varadarajulu et al., as the first in the world, described the multiple transluminal gateway technique (MTGT) in 2011, which consisted of the execution of a few transmural fistulas between the lumen of the gastrointestinal tract and the lumen of the WOPN [20]. Varadarajulu et al. proved that the use of several (2-3) routes of access to the necrotic cavity (MTGT) is a more effective technique of endoscopic treatment than single transluminal access to necrosis (SGT) [20]. The authors reported therapeutic success in 11/12 (91.7\%) patients treated with MTGT in comparison to $25 / 48$ (52.1\%) patients who underwent SGT [12]. In 2014, Mukai et al. described a technique that enables access to extensive necrotic areas through a single fistula (SGTMD) without the need to create an additional transmural route [21]. The authors reported therapeutic success in all 5 (100\%) patients [21].

Single transluminal gateway technique is based on total removal of necrotic tissues through the single fistula created between the cavity of necrotic collection and the lumen of the gastrointestinal tract $[7,13,16]$. This technique is particularly efficient in the case of unilocular collections of necrosis. In most of cases, WOPN takes the form of multilocular space divided by septa. Furthermore, decompression of the necrotic cavity during interventional treatment results in the formation of separated necrotic areas that are in fact separate cavities (undrained areas) communicating with one another via narrow canals [21]. In such cases a single access (SGT) to necrotic collection is insufficient. An additional access to necrosis $[11,15]$ or the use of other endoscopic techniques (endoscopic necrosectomy, active transpapillary drainage, creation of another transmural access) is necessary [10, 17-23].

To the best of our knowledge, this is the only study in the literature introducing the results of treatment of WOPN with the application of single transluminal gateway transcystic multiple drainage performed on such a large group of patients.

The study has some limitations. The main limitations of this study are lack of randomisation, retrospective character, and highly select group of patients from a single centre.

The development of endoscopic methods of treatment for WOPN has significantly improved the efficiency of endotherapy and reduces the use of other minimally invasive techniques. Currently, endoscopic drainage is a common method of treatment for patients with symptomatic walled-off pancreatic necrosis. Endoscopic treatment is an alternative for other minimally invasive techniques of treatment of pancreatic necrosis. In more and more patients with consequences of acute necrotising pancreatitis, endotherapy can remain the only method of treatment.

\section{Conclusions}

Single transluminal gateway transcystic multiple drainage is an effective method of endoscopic treat- 
ment for extensive walled-off pancreatic necrosis, with an acceptable amount of complications. However, the method of interventional treatment of pancreatic necrosis depends not only on the location of necrosis, but also on the experience of the medical centre.

\section{Conflict of interest}

The authors declare no conflict of interest.

\section{References}

1. Stamatakos M, Stefanaki C, Kontzoglou K, et al. Walled off pancreatic necrosis. World J Gastroenterol 2010; 16: 1701-12.

2. Sarr MG. 2012 revision of the Atlanta classification of acute pancreatitis. Pol Arch Med Wewn 2013; 123: 118-24.

3. Banks PA, Bollen TL, Dervenis C, et al. Classification of acute pancreatitis - 2012: revision of the Atlanta classification and definitions by international consensus. Gut 2013; 62: 102-11.

4. da Costa DW, Boerma D, van Santvoort HC, et al. Staged multidisciplinary step-up management for necrotizing pancreatitis. Br J Surg 2014; 101: e65-79.

5. Cremer M, Deviere J, Engelholm L. Endoscopic management of cysts and pseudocysts in chronic pancreatitis: long-term follow-up after 7 years of experience. Gastrointest Endosc 1989; 35: 1-9.

6. Grimm H, Binmoeller KF, Soehendra N. Endosonography-guided drainage of a pancreatic pseudocyst. Gastrointest Endosc 1992; 38: 170-1.

7. Baron TH, Thaggard WG, Morgan DE, Stanley RJ. Endoscopic therapy for organized pancreatic necrosis. Gastroenterology 1996; 111: 755-64.

8. Baron TH, Harewood GC, Morgan DE, Yates MR. Outcome differences after endoscopic drainage of pancreatic necrosis, acute pancreatic pseudocysts, and chronic pancreatic pseudocysts. Gastrointest Endosc 2002; 56: 7-17.

9. Sadik R, Kalaitzakis E, Thune A, et al. EUS-guided drainage is more successful in pancreatic pseudocysts compared with abscesses. World J Gastroenterol 2011; 17: 499-505.

10. Smoczyński M, Jagielski M, Jabłońska A, Adrych K. Endoscopic necrosectomy under fluoroscopic guidance - a single center experience. Videosurgery Miniinv 2015; 10: 237-43.

11. Szeliga J, Jackowski M. Minimally invasive procedures in severe acute pancreatitis treatment - assessment of benefits and possibilities of use. Videosurgery Miniinv 2014; 9: 170-8.

12. Šileikis A, Pečiulytė E, Misenkienė A, et al. Is minimally invasive surgical treatment justified for severe acute necrotizing pancreatitis patients with dysfunction of two or more organ systems? Videosurgery Miniinv 2017; 12: 225-30.

13. Smoczyński M, Marek I, Dubowik M, et al. Endoscopic drainage/ debridement of walled-off pancreatic necrosis - single center experience of 112 cases. Pancreatology 2014; 14: 137-42.

14. Papachristou GI, Takahashi N, Chahal P, et al. Peroral endoscopic drainage/debridement of walled-off pancreatic necrosis. Ann Surg 2007; 245: 943-51.

15. Ross AS, Irani S, Gan SI, et al. Dual-modality drainage of infected and symptomatic walled-off pancreatic necrosis: long-term clinical outcomes. Gastrointest Endosc 2014; 79: 929-35.
16. Jagielski M, Smoczyński M, Jabłońska A, et al. The role of endoscopic ultrasonography in endoscopic debridement of walled-off pancreatic necrosis - a single center experience. Pancreatology 2015; 15: 503-7.

17. Smoczyński M, Jagielski M, Jabłońska A, Adrych K. Transpapillary drainage of walled-off pancreatic necrosis - a single center experience. Videosurgery Miniinv 2015; 10: 527-33.

18. Seifert H, Biermer M, Schmitt W, et al. Transluminal endoscopic necrosectomy after acute pancreatitis: a multicentre study with long-term follow-up (the GEPARD Study). Gut 2009; 58 : 1260-6.

19. van Brunschot S, Fockens P, Bakker OJ, et al. Endoscopic transluminal necrosectomy in necrotising pancreatitis: a systematic review. Surg Endosc 2014; 28: 1425-38.

20. Varadarajulu S, Phadnis MA, Christein JD, Wilcox CM. Multiple transluminal gateway technique for EUS-guided drainage of symptomatic walled-off pancreatic necrosis. Gastrointest Endosc 2011; 74: 74-80.

21. Mukai S, Itoi T, Sofuni A, et al. Novel single transluminal gateway transcystic multiple drainages after EUS-guided drainage for complicated multilocular walled-off necrosis (with videos). Gastrointest Endosc 2014; 79: 531-5.

22. Mukai S, Itoi T, Sofuni A, et al. Expanding endoscopic interventions for pancreatic pseudocyst and walled-off necrosis. J Gastroenterol 2015; 50: 211-20.

23. Jagielski M, Smoczyński M, Adrych K. Endoscopic treatment of multilocular walled-off pancreatic necrosis with the multiple transluminal gateway techniques. Videosurgery Miniinv 2017; 12: 199-205.

Received: 11.02 .2018

Accepted: 7.06 .2018 